\title{
La création en acte. Devenir de la critique génétique, sous la direction de Paul Gifford et Marion Schmid
}

\section{Gabriella Bosco}

\section{(2) OpenEdition}

1 Journals

\section{Edizione digitale}

URL: http://journals.openedition.org/studifrancesi/9074

DOI: 10.4000/studifrancesi.9074

ISSN: 2421-5856

\section{Editore}

Rosenberg \& Sellier

\section{Edizione cartacea}

Data di pubblicazione: 1 octobre 2008

Paginazione: 503-504

ISSN: 0039-2944

\section{Notizia bibliografica digitale}

Gabriella Bosco, «La création en acte. Devenir de la critique génétique, sous la direction de Paul Gifford et Marion Schmid», Studi Francesi [Online], 155 (LII | II) | 2008, online dal 30 novembre 2015, consultato il 12 janvier 2021. URL: http://journals.openedition.org/studifrancesi/9074 ; DOI: https://doi.org/ 10.4000/studifrancesi.9074

Questo documento è stato generato automaticamente il 12 janvier 2021.

\section{(c) $($ ) $\odot$}

Studi Francesi è distribuita con Licenza Creative Commons Attribuzione - Non commerciale - Non opere derivate 4.0 Internazionale. 


\title{
La création en acte. Devenir de la critique génétique, sous la direction de Paul Gifford et Marion Schmid
}

\author{
Gabriella Bosco
}

\section{NOTIZIA}

La création en acte. Devenir de la critique génétique, sous la direction de Paul GIFFORD et Marion SCHMID, «Faux titre» 289, Amsterdam-New York, Rodopi, 2007, pp. 309.

1 La critica genetica, "invenzione" di un gruppo di specialisti dell'opera di Heine che, all'inizio degli anni Settanta, fondarono il Centre d'analyse des manuscrits modernes oggi diventato Institut des textes et manuscrits modernes (ITEM), è una disciplina ancora giovane ma già fortemente rappresentata in Europa come negli Stati Uniti. Le resistenze incontrate nella sua ormai quasi quarantennale esistenza da parte di ambienti lontani come quello dei filologi classici, che vissero la nascita della critica genetica come un'usurpazione dei loro territori, o quello dei sociologi, che l'accusarono di promuovere un recupero del positivismo più tradizionale nel settore degli studi letterari, hanno in realtà contribuito a rafforzare la nuova disciplina, obbligando i généticiens a mettere a punto una rigorosa concettualizzazione dei suoi fondamenti teorici e metodologici.

Il volume curato da Paul GIFFORD e Marion SCHMID risponde a una duplice esigenza: da un lato, fare un bilancio dei trentacinque anni di vita della critica genetica; d'altro lato, illustrare il panorama delle sue possibilità per il futuro. Con l'intento quindi di enucleare la specificità degli studi genetici rispetto agli altri approcci critici del testo, $\mathrm{e}$ di indagare la potenzialità di rinnovamento della critica letteraria che la genetica racchiude, i più eminenti genetisti si sono riuniti a Londra nel 2003 per un convegno internazionale - «Genèse, (inter)texte, création: où en sont les études génétiques» - $\mathrm{i}$ cui atti, qui raccolti, riassumono i grandi dibattiti sorti all'interno e alle frontiere della 
disciplina, confrontano casi particolari di genesi e tentato di fare il punto sulle acquisizioni della genetica per la teoria e la pratica della letteratura.

3 I contributi sono organizzati in cinque capitoli. Il primo, «Les études génétiques aujourd'hui et demain», si apre con un articolo di sintesi a firma del fondatore dell'ITEM, Louis HAY (che ha poi diretto l'Istituto fino al 1985): Critique génétique et théorie littéraire: quelques remarques (pp. 13-27). Almuth GRÉSILLON prosegue il discorso tornando, a dieci anni dal suo importante saggio Eléments de critique génétique, sulle definizioni fornite allora e rilanciando la questione delle frontierre della critica genetica («Nous avançons toujours sur des sables mouvants». Espaces et frontières de la critique génétique, pp. 29-40). Joseph JURT, sociologo della letteratura, indaga in particolare la frontiera che la critica genetica e una generale genetica delle società hanno in comune. Il suo intervento (Génétique textuelle et génétique sociale, pp. 41-50) si pone sulla scia della teoria del campo letterario di Pierre Bourdieu. E William MARX chiude la prima sezione interrogandosi su Les résistences théoriques à la critique génétique (pp. 51- 63).

4 La seconda parte, intitolata "Le chantier génétique», esemplifica il lavoro dei genetisti applicato alle pratiche di scrittura di cinque grandi scrittori del XIX e XX secolo: Eric LE CALVEZ dà una dimostrazione di genetica delle scene occupandosi della celebre passeggiata in carrozza di Madame Bovary (Génétique scénarique: les scénarios de la scène du fiacre dans "Madame Bovary", pp. 67-82); Nathalie MAURIAC DYER riflette sulla questione della struttura intenzionale di un testo incompiuto a partire dalla riscrittura proustiana del penultimo volume della Recherche (Proust entre deux textes: réécriture et «intention» dans "Albertine disparue», pp. 83-96); David NOTT affronta da un punto di vista psicoanalitico La difficile gestation de "La Truite" de Roger Vaillant (pp. 97-109); e Brian STIMPSON si occupa della dinamica degli inizi nella scrittura di Valéry e Marguerite Duras, dimostrando come in entrambi gli scrittori - pur così diversi - motore della scrittura sia il senso di una mancanza (Au commencement fut la fin: l'écriture en devenir chez Valéry et Duras, pp. 111-129).

5 «Hypertexte/Hypermédia», la terza sezione, illustra gli effetti della rivoluzione informatica sullo studio, l'edizione e la diffusione dei manoscritti moderni. Thomas BARTSCHERER, membro dell'équipe «HyperNietzsche», esemplifica il discorso parlando dello strumento di ricerca in rete che ha radicalmente innovato gli studi nitzscheani; Tony wiLliams dimostra i vantaggi dell'ipertesto nell'approccio della scrittura di Flaubert (Avant-texte, intertexte, hypertexte: l'épisode du Club de l'Intelligence dans "L'éducation sentimentale", pp. 159-169); mentre Domenico FIORAMONTE e Cinzia PUSCEDDU spiegano l'evoluzione del concetto di "testo" in seguito alla comparsa di nuovi supporti di comunicazione (Temps, texte, machines. Représenter le processus d'écriture sur le Web, pp. 171-188); e Pascal michelucci esamina l'apporto che il concetto di "virtuale" ha rappresentato per la critica genetica (La création virtuelle, pp. 189-201).

6 Nella quarta parte, «Enjeux de l'écriture, enjeux théoriques: penser la création?», torna a venir posto, alla luce delle recenti acquisizioni, il problema della concettualizzazione della genesi letteraria dal punto di vista teorico come da quello pratico. Daniel FERRER si chiede se e come la prospettiva genetica possa modificare la nozione di intertestualità (Quelques remarques sur le couple intertextualité-genèse, pp. 205-216). Paul GIFFORD invita a considerare la genetica una derivazione dell'ermeneutica, disciplina che sul piano epistemologico gli pare ben più adatta a comprendere e spiegare il lavoro dei genetisti di quanto non lo sia la "decostruzione" di discendenza idealista e razionalista 
(L'herméneutique et la création en acte, pp. 217-233). Robert PICKERING invece, illustrando le possibilità euristiche della genetica, ne immagina il futuro come disciplina di ricerca letteraria (La génétique entre singularité et pluralité de ses possibles heuristiques, pp. 235-251). L'ultimo contributo di questa quarta sezione consiste nella testimonianza della romanziera Marie DARRIEUSSECQ che, conversando con Jean-Marc TERRASSE, spiega come ha concepito e scritto il suo Truismes, romanzo del 1996 diventato best-seller internazionale, evocando il rapporto tra il testo in divenire e l'inconscio («Comment j'écris», pp. 253-268).

7 Il capitolo conclusivo del volume, «L'œuvre, l'écriture, la création: vocation et avenir des études génétiques», contiene la trascrizione della tavola rotonda che chiudeva il convegno di Londra, nel corso della quale genetisti e critici letterari discussero chiedendosi se la critica genetica possa comportare un rinnovamento del modo di guardare il testo letterario (Les études de genèse renouvellent-elles notre regard sur le texte littéraire?, pp. 269-290). 